\title{
Myeloma cells inhibit osteogenic differentiation of mesenchymal stem cells and kill osteoblasts via TRAIL-induced apoptosis
}

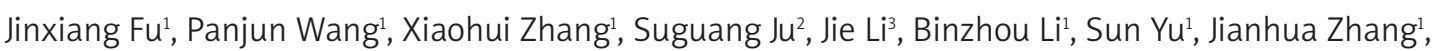
Xueguang Zhang

\author{
1Department of Haematology, No. 2 Affiliated Hospital of Soochow University, Suzhou, \\ Jiangsu Province, China \\ 2Institute of Biotechnology, Soochow University, China \\ 3Department of Haematology, Affiliated Children's Hospital of Soochow University, \\ Suzhou, Jiangsu Province, China
}

Submitted: 30 July 2009

Accepted: 15 September 2009

Arch Med Sci 2010; 6, 4: 496-504

DOI: 10.5114 /aoms.2010.14459

Copyright (c) 2010 Termedia \& Banach

\section{Abstract}

Introduction: Myeloma bone disease (MBD) is the result of the increased activity of osteoclasts (OCS), which is not accompanied by a comparable increase of osteoblast (OB) function, thus leading to enhanced bone resorption. Osteoblasts can also regulate osteoclast activity through expression of cytokines, such as receptor activator of nuclear factor- $\mathrm{KB}$ ligand (RANKL), which activates osteoclast differentiation, and osteoprotegerin (OPG), which inhibits RANKL by acting as a decoy receptor.

Material and methods: Based on a series of 21 patients with multiple myeloma (MM) and human osteoblast cell line HFOB1.19, we provide evidence that the bone marrow-derived mesenchymal stem cells (BMMSCs) of patients with MM exhibit normal phenotype, but showed reduced efficiency to differentiate into OBs as compared with normal controls.

Results: In vitro assays showed that MM cells inhibited the potential of osteogenic differentiation of BMMSCs from healthy controls and rendered the OBs sensitive to TRAIL-induced apoptosis. There was no evidence of the formation of tartrateresistant acid phosphatase positive OCs. The osteogenic differentiation of HFOB1.19 was also inhibited in the presence of RPMI 8266 or XG7 MM cells, as confirmed by von Kossa and ALP staining. Osteoblast s induced from BMMSCs supported survival and proliferation of MM cells, especially when the MM cells were cultured in medium containing rhTRAIL and dexamethasone. Multiple myeloma cells proliferated and grew well in the presence of residual OBs.

Conclusions: Besides OCs, our results demonstrated that OBs and MM cells were dependent upon each other and made a microenvironment suitable for MM cells.

Key words: multiple myeloma, osteoblast, recombinant human tumour necrosis factor (TNF)-related apoptosis-inducing ligand (TRAIL), myeloma bone disease (MBD).

\section{Introduction}

Multiple myeloma ( $\mathrm{MM}$ ) is the most common form of plasma cell dyscrasia, affecting $B$ cells that have traversed the post-germinal centre.

\author{
Corresponding author: \\ Dr. Jinxiang Fu \\ Haematology Department \\ No. 2 Affiliated Hospital \\ of Soochow University \\ Suzhou 215004 \\ Jiangsu Province, China \\ Phone: 8651267784066 \\ Fax: 8651268665552 \\ E-mail: \\ uufjxly@public1.sz.js.cn
}


Plasma cells in MM first enter blood vessels in the peripheral blood, from where they eventually migrate to, survive in and expand exclusively in the bone marrow. At advanced stages, myeloma cells can grow independently of the bone marrow microenvironment, causing a more aggressive presentation of the disease, which includes mainly lytic lesions, pathological fractures, hypercalcaemia and osteoporosis [1-3]. Myeloma bone disease (MBD) is the result of the increased activity of osteoclasts, which is not accompanied by a comparable increase of osteoblast function, thus leading to enhanced bone resorption [4, 5]. Osteolytic lesions in multiple myeloma are only found adjacent to intramedullary plasma cell foci or plasmacytomas, suggesting that MM cells may secrete factors that promote activation of osteoclasts, and inactivation of osteoblast function to replace the bone loss. This peculiar feature of $M B D$ is sustained by the extreme paucity of osteocytes or newly formed bone within the erosion sites. Enhanced bone resorption, however, is not accompanied by concurrent enhancement even in patients achieving chemotherapy-induced complete remission [6]. Osteoblasts originate mainly from mesenchymal stem cells (MSCs) and are responsible for bone matrix synthesis by secreting collagen, which form strands called osteoids [7]. Osteoblasts can also regulate osteoclast activity through expression of cytokines, such as RANKL, which activates osteoclast differentiation, and osteoprotegerin (OPG), which inhibits RANKL by acting as a decoy receptor [8]. Investigation of the reduction of osteoblast activity in $M M$ has been overshadowed by the volume of work focused on the role of hyperactivated osteoclasts [9].

Tumour necrosis factor (TNF)-related apoptosisinducing ligand (TRAIL), first discovered in 1995, is a member of the TNF superfamily that induces apoptosis through engagement of death receptors. TRAIL induces apoptosis in numerous types of human tumours and rodent xenograft models [10], including MM [11-13], while exerting minimal or no toxicity in normal cells. TRAIL interacts with a complex system of receptors in human cells: 2 proapoptotic death receptors (DR4 and DR5), which have cytoplasmic death domains, and 2 decoy receptors, which are functionally inactive (DCR1 and DcR2).

Besides tumour control by TRAIL ${ }^{+}$immune cells, there is accumulating evidence that death receptor ligands might also be used by tumour cells themselves to kill bystander cells in a mechanism called "tumour counterattack" [14-16]. The mechanisms involved in the pathogenesis of MBD are not fully elucidated, though the prevalent opinion is that malignant plasma cells exert a major osteoclastogenic effect by promoting the recruitment, differentiation and activation of osteoclast progenitor within the bone marrow following their interaction with the MSCs. In the present study, we tried to elucidate whether the formation of bone lesions that develop with high frequency in MM patients is mediated, at least in part, by interaction of myeloma cells and osteoblasts either through TRAIL or by inhibiting osteoblastic potential of mesenchymal stem cells, thereby inducing cell death in the bone cell fraction. Moreover, effects of osteoblasts on proliferation and survival of MM cells, especially in medium containing dexamethasone or with TRAIL, were also investigated using a co-culture technique in vitro.

\section{Material and methods}

\section{Culture of mesenchymal stem cells derived from bone marrow}

Twenty-one patients with newly diagnosed MM, whose median age was 56 years (range 31-74 years), were studied before any treatment with informed consent. Among these patients, 3 were in stage I or II of the disease and the rest were in stage III. Mononuclear cells (MNCs) were separated from bone marrow of patients mentioned above and normal controls by Ficoll-Hypaque density gradient centrifugation. To remove haematopoietic stem cells and prevent overgrowth of the cultures with macrophages, $\mathrm{CD} 45^{+}$cells were depleted by negative immunomagnetic cell selection using the Mini MACs device (Miltenyi Biotec, Germany) according to the manufacturer's instructions. Samples obtained before and after depletion of the CD45 $5^{+}$cells were analysed by flow cytometry (FCM). The resulting CD45-depleted MNCs were cultured as described previously [17]. When adherent cells (primary culture, $\mathrm{P} 0$ ) reached $80 \%$ confluence, cells were isolated by treatment with $0.25 \%$ trypsin (Invitrogen, USA)/EDTA (Sigma, USA) and cultured immediately at $10^{3}$ cells $/ \mathrm{cm}^{2}$ under the same conditions (first passage, P1). BMMSCs at P0 and P1 were used immediately or frozen until use.

\section{Culture of human osteoblast cell line HFOB1.19}

The human osteoblast cell line HFOB1.19 was bought from Shanghai Cell Bank, Academy of Cell Science, China. According to their instructions, the cells were cultured at $2 \times 10^{5} / \mathrm{ml}$ in $\alpha-M E M+10 \%$ fetal calf serum (FCS). Then the cells were fed twice a week until confluence. HFOB1.19 cells were detached using $0.25 \%$ trypsin and counted using trypan blue exclusion. HFOB1.19 cells were used after expansion in vitro or frozen until use.

\section{Flow cytometry analysis}

The phenotypes of both BMMSCs and HFOB1.19 were determined by flow cytometry (FCM) with 
FITC- or phycoerythrin-conjugated anti-CD90, CD106, CD44, CD45, CD13, CD14, CD73, anti-human TRAIL-R1, TRAIL-R2 and anti-human TRAIL monoclonal antibodies (all from Invitrogen, USA). Expression of TRAIL of MM cell lines RPMI 8266 (ATCC, USA) and XG7 (gratefully donated by Prof. Zhang Xueguang, Institute of Biotechnology, Soochow University) was also analysed with FCM. Samples were analysed on an EPICS-ALTRA flow cytometer (Beckman Coulter, USA) using EXPO2 software. Expression level of each molecule was quantified as the mean fluorescence intensity (MFI).

\section{Differentiation of bone marrow-derived mesenchymal stem cells into osteoblasts}

Bone marrow-derived mesenchymal stem cells (P0 or P1) and HFOB1.19 were seeded alone at $104 / \mathrm{cm}^{2}$ in 6 -well culture plates in DMEM with $4.5 \mathrm{~g} /$ g glucose supplemented with 10\% FCS, $0.1 \mu \mathrm{M}$ dexamethasone and $50 \mu \mathrm{g} / \mathrm{ml}$ ascorbic acid (Sigma, USA). The MM cell line RPMI 8266 or XG7 precultured in the same culture medium was added to the cultures above at a dose of $10^{5} \mathrm{cell} / \mathrm{ml}$ at the beginning of culture and at day 7. Culture medium was changed twice a week. After 3 weeks' incubation, the culture was washed with phosphate-buffered saline (PBS) and stained for alkaline phosphatase (ALP) and von Kossa using a commercial assay kit (Protein Assay, USA). To exclude the possible effects of osteoclasts in a coculture manner, tartrate-resistant acid phosphatase (TRAP, Sigma, USA) staining was used at the same time.

Role of TRAIL on proliferation of HFOB1.19 and bone marrow-derived mesenchymal stem cells in vitro

To determine whether TRAIL has a proliferative or apoptotic effect on osteoblasts, the HFOB1.19 cells were cultured at a density of $2 \times 10^{3} / \mathrm{ml}$ in 96 and 6-well plates in $\alpha-M E M+10 \%$ FCS with $25 \mathrm{ng} / \mathrm{ml}, 50 \mathrm{ng} / \mathrm{ml}, 100 \mathrm{ng} / \mathrm{ml}$ and $200 \mathrm{ng} / \mathrm{ml}$ rhTRAIL (Invitrogen, USA). After incubation for $24 \mathrm{~h}$, cell viability was determined with the MTT assay and was expressed as percentage of the corresponding values for control cells. The percentages of apoptotic HFOB1.19 cells were determined with the annexin V/PI assay. The role of TRAIL on osteoblasts induced from BMMSCs was also investigated with the same method with TRAIL at a concentration of $50 \mathrm{ng} / \mathrm{ml}$.

\section{Multiple myeloma cells and osteoblast co-cultures}

For determination of cytotoxic activity of myeloma cells on osteoblasts, we performed direct and indirect co-culture experiments.
1) Direct contacted groups: (1) RPMI 8266 or $X G 7$ was cultured in $1 \mathrm{ml}$ of $\alpha$-MEM with $50 \mathrm{ng} / \mathrm{ml}$ rhTRAIL without osteoblasts; (2) the osteoblasts or HFOB1.19 were cultured with XG7 or PRMI $8266 \mathrm{MM}$ cells in $1 \mathrm{ml}$ of $\alpha$-MEM with $50 \mathrm{ng} / \mathrm{ml}$ rhTRAIL; (3) the osteoblasts and HFOB1.19, which were pre-incubated with XG7 or PRMI 8266 MM cells for $24 \mathrm{~h}$, were cultured in medium containing $50 \mathrm{ng} / \mathrm{ml}$ rhTRAIL; (4) the osteoblasts and HFOB1.19 were cultured with XG7 or PRMI 8266 MM cells without rhTRAIL; (5) the osteoblasts and HFOB1.19 were cultured with XG7 or PRMI $8266 \mathrm{MM}$ cells with $10 \mathrm{\mu g} / \mathrm{ml}$ anti-TRAILR1/R2 mab.

2) Indirect contacted groups: Transwell $(0.45-\mu \mathrm{m}$ pore size, Corning Co, USA) culture system was used in this group according to the manufacturer's instructions. (1) The osteoblasts and HFOB1.19 in the lower chamber and RPMI8266(XG7) in the upper chamber with $50 \mathrm{ng} / \mathrm{ml}$ rhTRAIL; (2) the osteoblasts and HFOB1.19 in the lower chamber and RPMI8266(XG7) in the upper chamber without rhTRAIL; (3) the osteoblasts and HFOB1.19 in the lower chamber and RPMI8266(XG7) in the upper chamber with $10 \mu \mathrm{g} / \mathrm{ml}$ anti-TRAIL-R1/R2 mab. The percentages of apoptotic BMMSCs, HFOB1.19, and MM cells were determined by the annexin V/PI assay.

\section{Effects of osteoblasts on multiple myeloma cells}

Bone marrow-derived mesenchymal stem cells and HFOB1.19 were seeded alone at $10^{4} / \mathrm{cm}^{2}$ in 24-well culture plates in osteoblastic-inducing medium as mentioned above for 14 days. The preculture cells were washed 3 times with PBS to detach and remove the non-adherent cells. RPMI8266 and XG7 MM cells $\left(5 \times 10^{5}\right.$ cells $/ \mathrm{ml}$ in $\alpha-M E M$ medium) were cultured alone or added to resulting osteoblasts in vitro. Culture medium was changed twice a week. At the end of day 3, 7, 10 and 14, MM cells were counted. To test the effects of OBs on survival of MM cells, RPMI8266 and XG7 MM cells were plated to 6-well plates at a concentration of $5 \times 10^{5} /$ well alone or containing freshly prepared OBs in medium with $2 \times 10^{-7} \mathrm{M}$ dexamethasone or $50 \mathrm{ng} / \mathrm{ml}$ rhTRAIL. The cultures were continued for $48 \mathrm{~h}$ and cell numbers, viability (trypan blue assay) and annexin V/PI binding were determined by flow cytometry.

\section{Data procedure}

Unless otherwise indicated, all values are expressed as mean \pm SE. Statistical analysis was performed using SPSS software (Chicago, IL). Statistical significance was determined using the nonparametric Mann-Whitney U test or Student's $t$ test. A value of $p<0.05$ was considered significant. 


\section{Results}

Morphological characteristics of in vitrocultured bone marrow-derived mesenchymal stem cells and flow cytometric data

Human BMMSCs were cultured as described in the Material and methods section. As early as $2 \mathrm{~h}$ post-incubation, CD45-depleted MNCs began to adhere to the wells and attachment increased with time. The round and stellate cells began to form small colonies after approximately 5 days in culture and CFU-F were fully established by day 7-10 (Figure 1).
Confluent BMMSC cultures were obtained from 20 out of 21 patients and all controls after 14 and 13.5 days, respectively. There was no difference between the cells from MM and normal controls with respect to their morphological characteristics, number of generated BMMSCs per $\mathrm{cm}^{2}$ and patient age. In the cases, BMMSC phenotypes were analysed at passage 2. The BMMSCs from patients or normal controls displayed similar antigen expression $(p>0.05$ for each antigen tested) with no expression of haematopoietic lineage antigens such as CD34, CD45 and CD14 and high expression of CD44, CD106, CD73, CD90 and
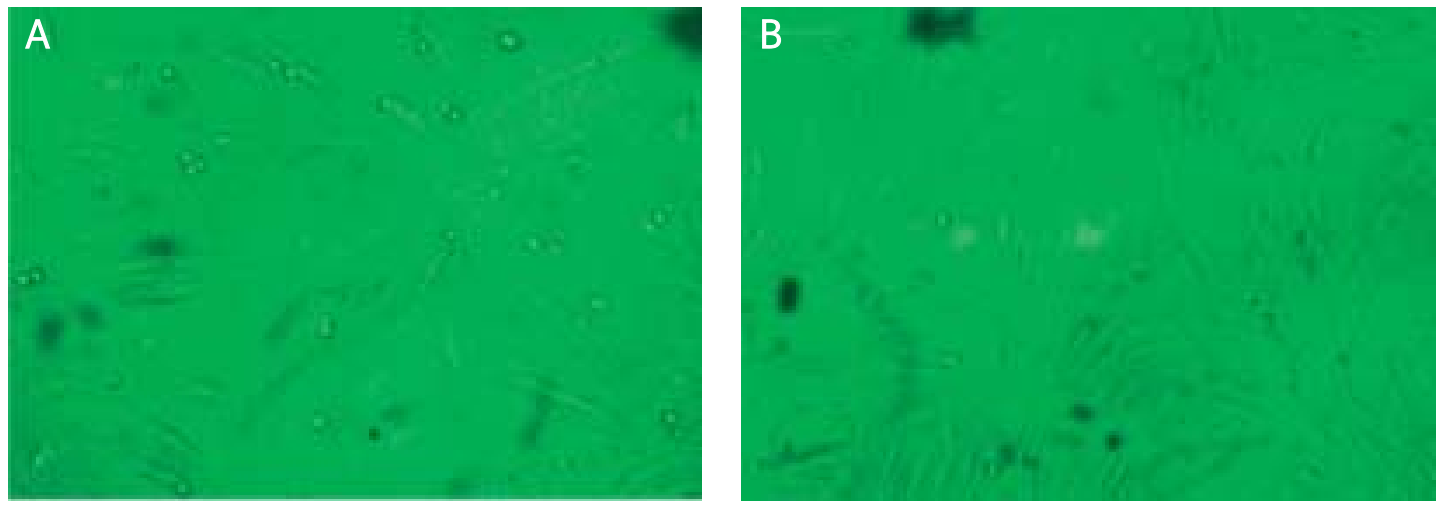

Figure 1. Morphological characterization of BMMNCs. A - BMMNCs cultured for 5 days, B - BMMNCs at day 12 (100x)
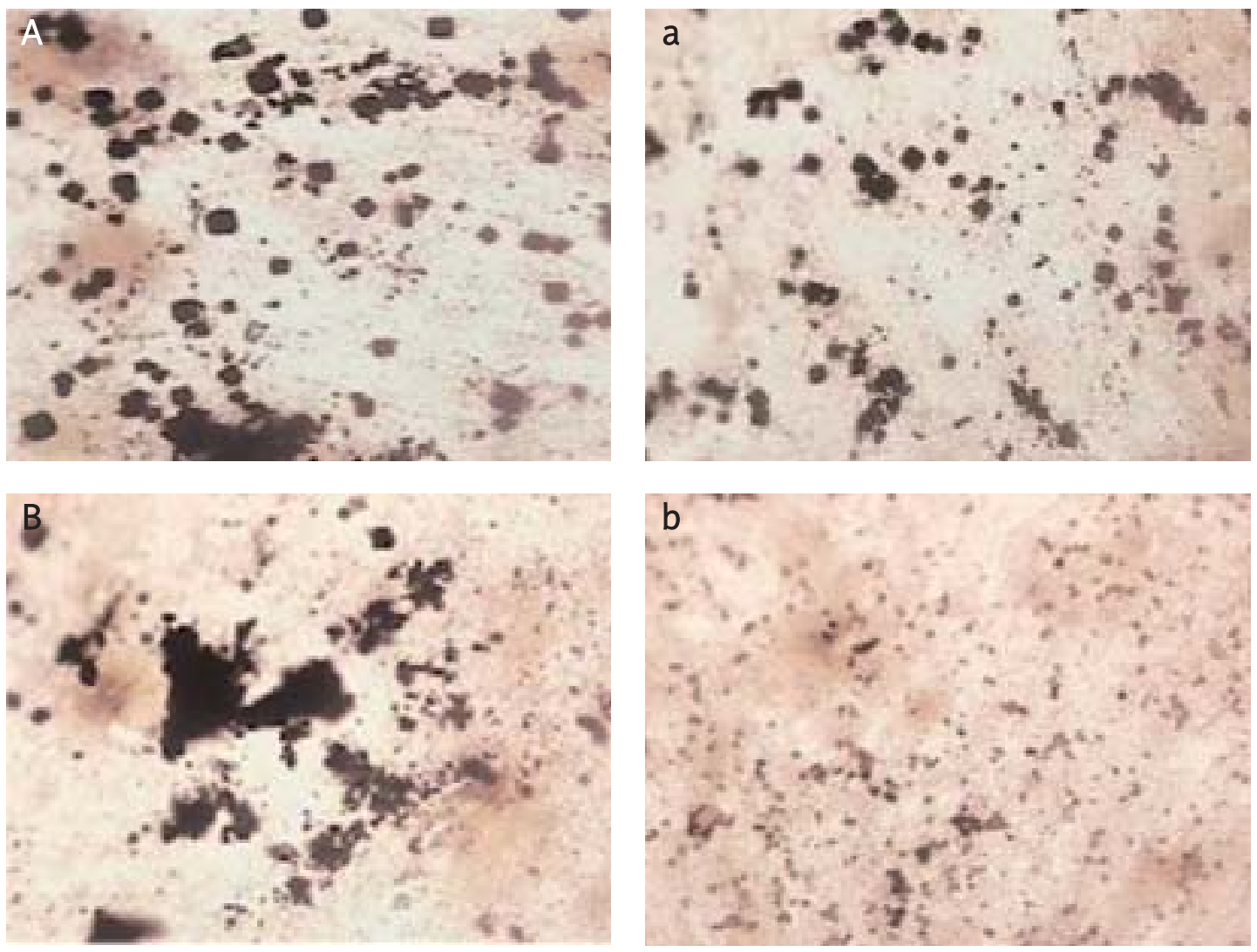

Figure 2. von Kossa staining mineralized crystal generated from BMMSCs. A and a - from normal controls and MM patients respectively after 21 days incubation with osteogenic inducing medium (100x). B and b-from normal controls and MM patients respectively after incubation with osteogenic condition medium and RPMI 8266 
CD13, while it is confirmed by FCM that HFOB1.19 only expressed CD44 and CD90 out of the above antigens. The studies also demonstrated that HFOB1.19 moderately expressed TRAIL-R2 and did not express TRAIL-R1, but we failed to detect either TRAIL-R1 or TRAIL-R2 expression on BMMSCs.

\section{Multiple myeloma cells inhibit the osteogenic differentiation of bone marrow-derived mesenchymal stem cells in vitro}

When cultured in monolayer osteogenic conditions, the BMMSCs isolated from MM patients and healthy controls acquired an osteoblastic morphology demonstrated by deposition of a calciumrich mineralized extracellular matrix (Figure 2A). Mineralized crystal appeared in BMMSC cytoplasm after 2 weeks, and the amount and volume of crystal
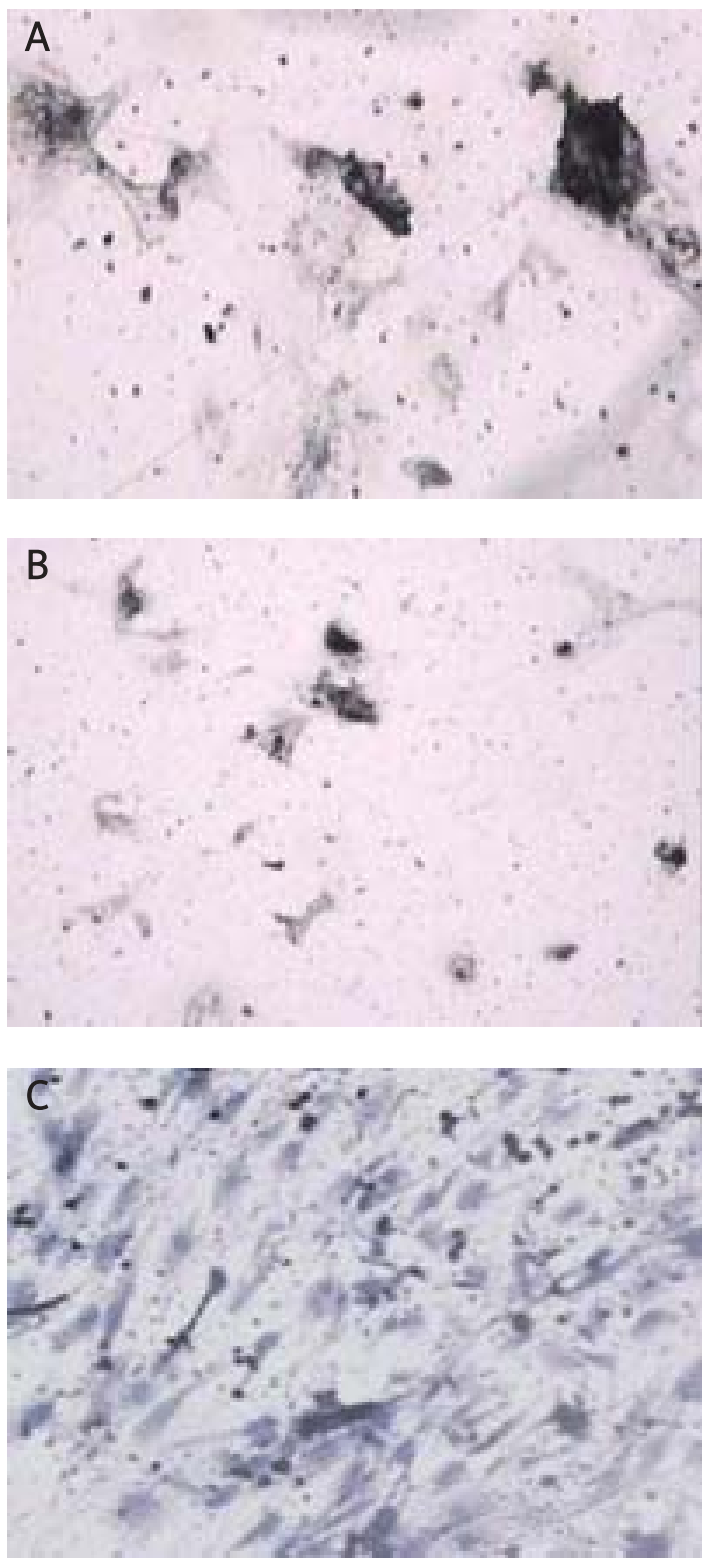

increased in a time-dependent manner. These findings suggested that osteogenesis of BMMSCs from MM patients were qualitatively conserved, but it seemed that the osteogenic differentiation of BMMSCS from MM patients was partially damaged as compared with the controls (Figure 2A and 2a). There were a few calcium-rich mineralized deposits and low activity of ALP in HFOB1.19 cells when stained with von Kossa and ALP, but accumulation of mineralized deposits and upregulation of ALP activity were seen when the cells were cultured in osteogenic conditions for 7 days. We then tested whether RPMI 8266 and/or XG7 MM cell lines can affect differentiation of $B M M S C s$ in vitro. The differentiation of BMMSCs or HFOB1.19 into osteoblast was inhibited in the presence of RPMI 8266 or XG7 whether the MM cells were plated at the beginning of culture or at 7 days (Figures 2, 3). The mineralized crystal deposits in the
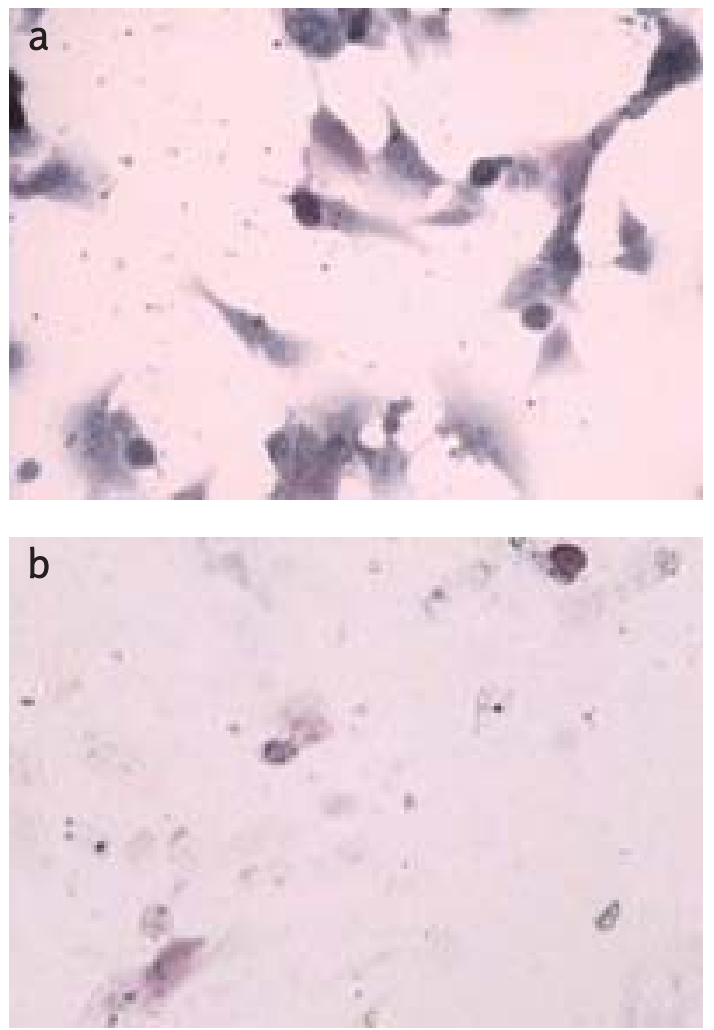

Figure 3. The change of ALP and mineralized deposition in HFOB1.19 before and after cells were cultured in osteogenic condition medium and RPMI 8266. Before the HFOB1.19 cells were incubated in osteogenic inducing medium with RPMI 8266, mineralized deposition and ALP activity were seen with von Kossa and ALP staining. A - von Kossa staining, a - ALP staining (100x). When the HFOB1.19 cells were incubated in osteogenic inducing medium with RPMI 8266 for 7 days, decreased of mineralized deposition and downregulation of ALP activity were seen as cells was stained with von Kossa and staining ( $B$ - von Kossa staining, $b$ - ALP staining $(100 \times), C$ - there was no TRAP positive found in coculture experiments 
cells decreased significantly in comparison with controls. There were no tartrate-resistant acid phosphatase (TRAP) positive cells found in our study (Figure 3C).

\section{Effects of TRAIL on osteoblasts}

After demonstrating with FCM that HFOB1.19 moderately expressed TRAIL-R2/DR5, we then investigated whether recombinant human TRAIL (rhTRAIL) would inhibit the growth of HFOB1.19 when the cells were cultured at different concentrations of TRAIL. HFOB1.19 was resistant to TRAIL-induced apoptosis when the cells were incubated for $24 \mathrm{~h}$ in the presence of rhTRAIL in vitro. Expression of TRAIL did not render HFOB1.19 sensitive to apoptosis as detected by FCM. Similar results were observed among the osteoblasts induced from BMMSCs (Figure 4).

\section{Myeloma cells rendered osteoblasts sensitive to TRAIL-induced apoptosis}

Since we observed inhibited osteogenic differentiation of BMMSCs in co-culture experiments with myeloma cells, we next addressed whether rhTRAIL would further increase cell death of osteoblasts when co-cultured with myeloma cells. We determined the total fraction of apoptotic osteoblasts using FCM. Osteoblasts or HFOB1.19 were resistant to TRAIL when cultured alone, while in co-culture with RPMI-8226 or XG7 cells a higher percentage of apoptotic osteoblasts was observed, which additionally increased following treatment with rhTRAIL. The pre-incubation of osteoblasts with MM cells rendered the osteoblasts sensitive to TRAIL-induced apoptosis, but the proportion of apoptotic cells decreased significantly as compared with co-culture. The fraction of apoptosis in osteoblasts induced by myeloma cells was significantly decreased when direct cell-cell contact was prevented with the Transwell culture system, suggesting that primarily membrane-bound factors are involved in myeloma-mediated cytotoxicity. The incubation of RPMI-8226 cells with anti-TRAIL-R1 mab was ineffective in blocking killing of osteoblasts, while the addition of anti-TRAIL-R2 mab significantly reduced the percentage of apoptotic osteoblasts. Similar results were seen when XG7 cells were used as effector cells (Figure 4C).

\section{Osteoblasts promote multiple myeloma cells to proliferate and survive TRAIL-induced apoptosis}

Pre-cultured OBs were incubated alone or cultured with RPMI 8266 or XG7 MM cells for $48 \mathrm{~h}$ in medium containing rhTRAIL or dexamethasone. The total number of myeloma cells recovered from cultures of myeloma cells alone was significantly
A

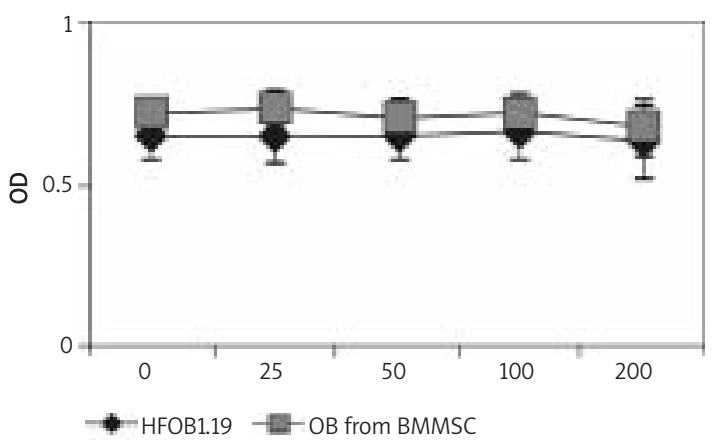

B

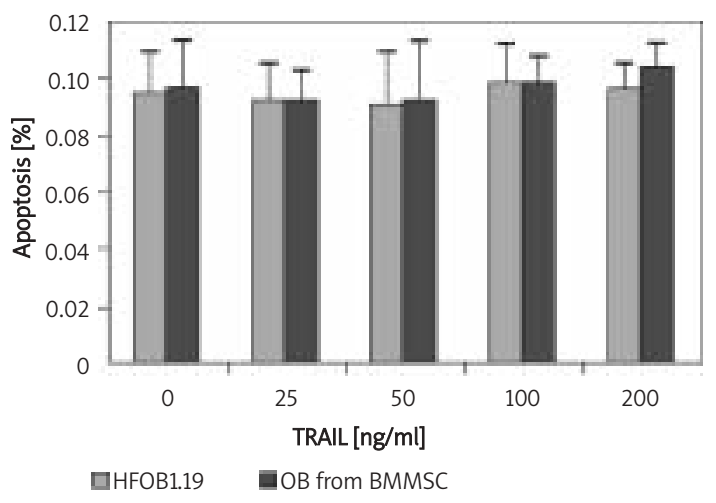

C

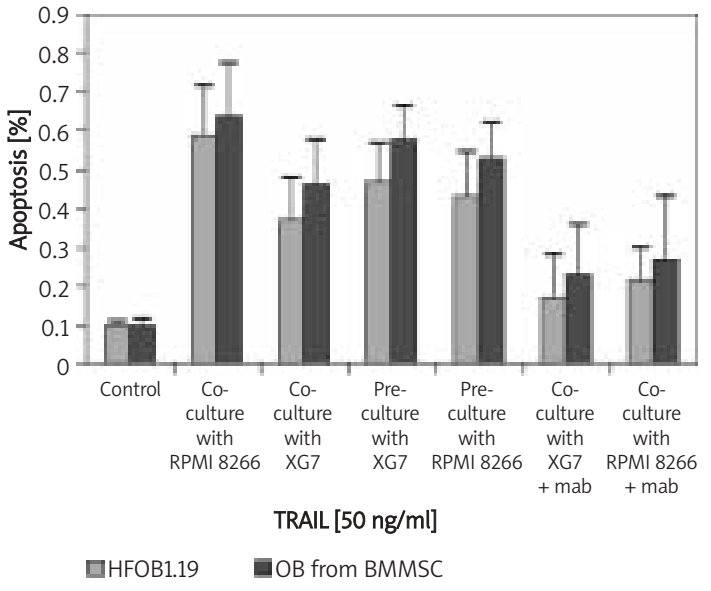

Figure 4. The OBs induced from BMMSCs or human osteoblast cell line HFOB1.19 grew well in medium containing rhTRAIL and were not sensitive to rhTRAIL induced apoptosis in vitro as MTT assays or flow cytometry using the annexin V/PI binding assay ( $A$ and $B$ ). The percentage of apoptosis increased significantly whether the OBs or HFOB1.19 were precultured or co-cultured with XG7/RPMI 8266 MM cells, but the proportion of apoptosis decreased significantly when anti-TRAIL-R2 mab were added to the cultured (C) 
lower than that from co-cultures and their viability was also significantly lower, while their apoptotic rate was significantly higher than that of myeloma
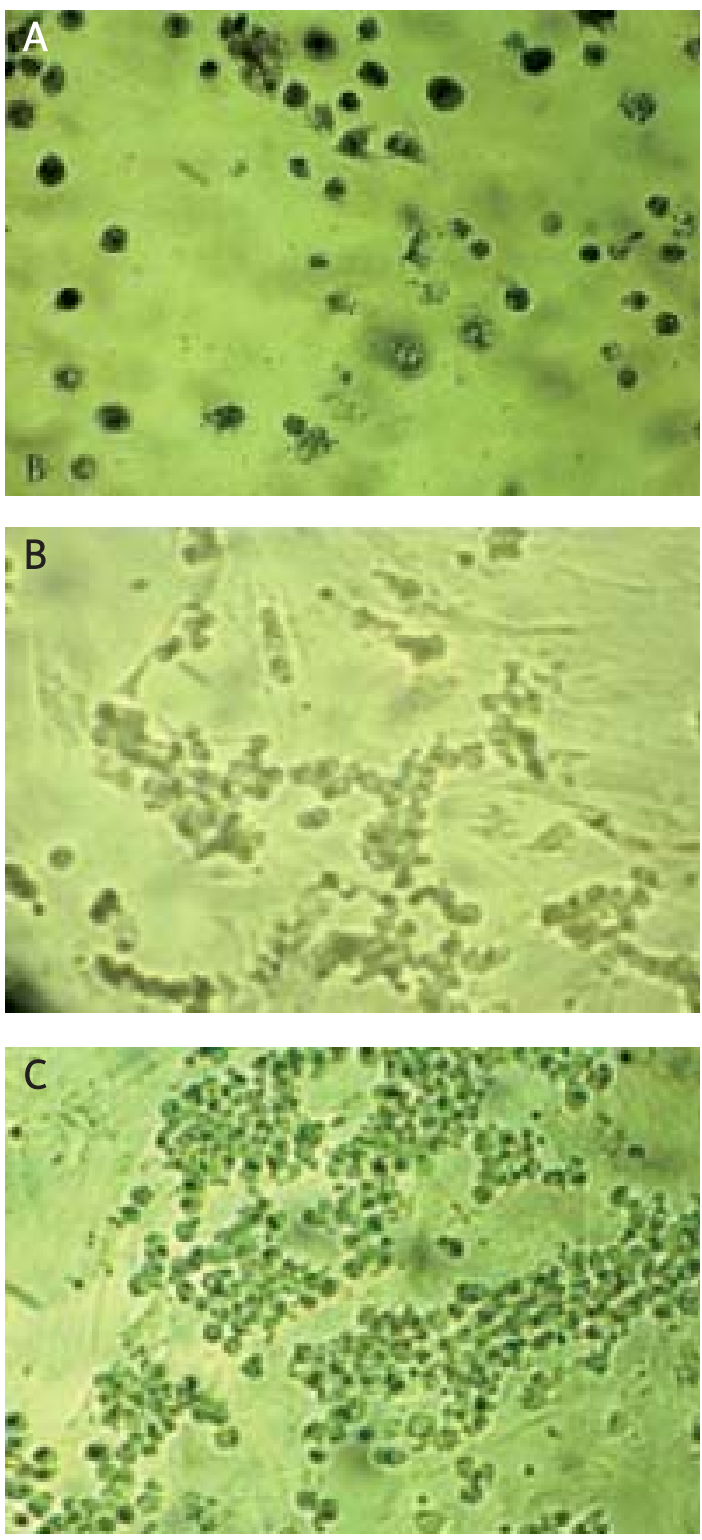

Figure 5. RPMI 8266 and XG7 MM cells were sensitive to TRAIL induced apoptosis (A), but the percentage of apoptosis decreased when the cells were co-cultured with osteoblasts (OBs); (B) MM cells and OBs were incubated in medium containg $2 \times 10^{-7} \mathrm{M}$ dexamethasone; (C) MM cells and OBs were incubated in medium containing $50 \mathrm{ng} / \mathrm{ml}$ rhTRAIL cells cultured with OBs (Table I, Figure 5). Our data also showed that OBs could promote MM cells to survive and grow in the presence of rhTRAIL and dexamethasone.

\section{Discussion}

Normal bone constantly undergoes remodelling, which involves the resorption of bone by osteoclasts and the deposition of new bone by osteoblasts at sites of previous resorption. In myeloma, many different components interact to increase the bone resorption process. These include myeloma cells themselves, bone marrow stromal cells, and $T$ cells present in the marrow microenvironment. Furthermore, growth factors released by the bone resorption process also increase the growth of myeloma cells. This creates a vicious cycle and finally induces increased bone resorption. This osteolytic bone disease is responsible for the most debilitating clinical symptoms of $M M$, which include bone pain, pathological fractures, spinal cord compression, hypercalcaemia, renal failure, and death [18-20]. Recently studies have been supported by clinical evidence showing that MM patients with advanced bone lesions may have a reduction of bone formation markers, such as alkaline phosphatase and osteocalcin, together with the increased bone resorption [21, 22]. Similarly, marked osteoblastopenia and reduced bone formation have also been reported in murine models of MM that develop bone lesions [23-25]. These data suggest that MM cells suppress osteoblasts and thereby inhibit bone formation. Wnt signalling pathway inhibitors DKK1 and sFRP-2 and soluble cytokines IL-3 and IL-7 were all reported to be inhibitors of bone formation in MM [26-28]. All of these studies employed bone marrow stromal cells, or pre-osteoblastic cell lines as the subjects. However, there is no report on the potential and regulation of osteogenesis of BMMSCs and interactions among the MM cells, BMMSCs and osteoblasts. Bone marrow-derived mesenchymal stem cells were the main source of osteoblasts in bone marrow and played a critical role in bone regeneration. We hypothesize that the impaired osteogenesis of MM patients may occur in stem cells more primitive than stromal or pre-osteoblastic cells, such as MSCs, and osteoblasts might promote $M M$ cell survival or growth in the special microenvironment of $\mathrm{MM}$ patients.

Table I. Effects of OBs on MM cells in medium containing rhTRAIL and dexamethasone

\begin{tabular}{|lcccccc|}
\hline & \multicolumn{3}{c}{ MM cells + rhTRAIL/Dex } & \multicolumn{3}{c|}{ MM cells + OBs + rhTRAIL/Dex } \\
\cline { 2 - 7 } & Annexin V [\%] & Annexin V/PI [\%] & Alive [\%] & Annexin V [\%] & Annexin V/PI [\%] & Alive [\%] \\
\hline 8226 & $18.08^{*} / 22.85^{*}$ & $54.07 / 62.84$ & $27.66^{*} / 14.14^{*}$ & $4.21^{*} / 17.14^{*}$ & $35.74 / 44.01$ & $57.71^{\# / 37.91^{*}}$ \\
\hline XG7 & $35.49^{*} / 16.81^{*}$ & $36.76 / 41.36$ & $26.24^{*} / 41.62^{*}$ & $4.32^{\#} / 16.95^{*}$ & $14.76 / 32.71$ & $80.92^{\# / 51.91^{*}}$ \\
\hline
\end{tabular}

${ }^{*} p<0.05,{ }^{*} p<0.01$ 
Arnulf et al. [29], demonstrated that MSCs isolated from MM expressed the typical expected phenotype and had haematopoiesis-supporting capacities similar to those of normal MSCs, but these cells produced on average 2.5 times more IL-6 than MSCs from normal controls and were less immunosuppressive than their normal counterparts. In this study, we isolated and purified the MSCs from BM of myeloma patients. Flow cytometry analysis of BMMSCs from patients and healthy controls displayed similar antigen expression with no expression of haematopoietic lineage antigens such as CD34, CD45 and CD14 and high expression of CD44, CD106, CD73, CD90 and CD13. Von Kossa staining showed that there were mineral deposits in both BMMSCs, which suggested that osteogenesis of BMMSCs from MM patients were qualitatively conserved, but it seemed that the osteogenic differentiation of BMMSCs from MM patients was partially impaired as compared with the healthy controls. Importantly, these characteristics were observed in the absence of $M M$ cells. We then tested whether RPMI 8266 and/or XG7 MM cell lines can affect differentiation of BMMSCs in direct contact manner in vitro. The differentiation of BMMSCs into osteoblasts decreased significantly in the presence of RPMI 8266 or XG7 whether the MM cells were plated at the beginning of culture or at day 7 . The mineralized crystal deposition in the cells observed was significantly less than that in controls. Lower crystal deposition and low ALP activity in HFOB1.19 were observed when the cells were incubated in the presence of $M M$ cells. Evidence is provided to support the existence of subsets of MM cells expressed in the osteoclast phenotype, including multinuclear morphology, cytoplasmic TRAP and a specific osteoclast antigen [30], which may contribute to MBD by producing osteoclast enzymes. However, neither the phenotype nor the functional properties of osteoclasts were detected and no TRAP positive cells were found in our study. These results suggest that the osteogenic potential of MSCs of MM was decreased and they support the notion that suppressed osteoblastic activity and low bone formation contribute to the bone disease found in MM patients.

It was reported recently that myeloma cell lines sensitized osteoblasts to cell death mediated by recombinant TRAIL, whereas primary osteoblasts protected myeloma cells from TRAIL-mediated apoptosis that was mediated by OPG [24]. Our results demonstrate that expression of TRAIL-R2 did not render HFOB1.19 sensitive to apoptosis induced by TRAIL as detected by FCM. Similar results were observed among the osteoblasts induced from BMMSCs. Neither OBs induced from BMMSCs nor HFOB1.19 would sensitize to TRAIL- induced apoptosis in vitro, but the proportion of apoptosis increased whether the cells were incubated with MM cells or pre-cultured with MM cells. We observed significant killing of osteoblasts in direct contact with MM cells and the fraction of apoptosis in osteoblasts induced by myeloma cells was significantly decreased when direct cell-cell contact was prevented with the Transwell culture system. The incubation of RPMI-8226/XG7 cells with anti-TRAIL-R1 mab was ineffective in blocking killing of osteoblasts, while the addition of anti-TRAIL-R2 mab significantly reduced the percentage of apoptotic osteoblasts. Vice versa, RPMI-8226 or XG7 cells were sensitive to treatment with rhTRAIL Their sensitivity, however, decreased when they were treated in co-culture with OBs. Interestingly, the residual OBs had the ability to support survival of MM cells in presence of dexamethasone or rhTRAIL. The proportion of apoptotic MM cells decreased significantly in comparison with controls. Although myeloma cells did not firmly adhere to OBs, cell-cell contact was essential, as demonstrated in non-contact experiments with OBs or OB/MM cell co-cultures. This indicates that soluble factors released by OBs alone or after their direct interaction with $M M$ cells are sufficient to promote myeloma cell growth, which may be parallel to the results of Shipman and Croucher [31]. In the meantime, it seemed that adhesive interactions between myeloma and BMMSCs played a significant role in the survival of MM cells and might augment the bone-destructive process. Based on these experiments, we hypothesized that primarily membrane-bound factors are involved in myeloma-mediated cytotoxicity and this process might allow them to gain space within the bone marrow necessary for their proliferation and expansion.

In conclusion, our BMMSC/myeloma cell coculture experiments clearly demonstrate that the osteogenic differentiation of BMMSC from MM patients was impaired, although the phenotype was similar to the healthy controls. Multiple myeloma cells inhibited the potential of osteogenic differentiation of BMMSCs from healthy controls and rendered the OBs sensitive to TRAIL-induced apoptosis. Osteoblasts from healthy donors supported survival and proliferation of MM cells, especially when the MM cells were cultured in medium containing rhTRAIL and dexamethasone. This might explain in part the lower number of OBs found from bone marrow of MM patients and making a microenvironment suitable for MM cells.

\section{Acknowledgments}

The authors thank Hong Zhang for technical assistance. This study was supported by grants from the Basic Scientific Research Programme 
Foundation of the Commission of Science Technology and Industry for National Defence (616010305) and Suzhou Social Development Foundation (SS0534).

\section{References}

1. Roux S, Meignin V, Quillard J, et al. RANK (receptor activator of nuclear factor-jB) and RANKL expression in multiple myeloma. Br J Haematol 2002; 117: 86-92.

2. Bataille R, Harousseau JL. Multiple myeloma. N Engl J Med 1997; 336: 1657-64.

3. Giuliani N, Colla S, Rizzoli V. New insight in the mechanism of osteoclast activation and formation in multiple myeloma: focus on the receptor activator of NF-kappaB ligand (RANKL). Exp Hematol 2004; 32: 685-91.

4. Joshua D, Brown R, Ho PJ. Multiple myeloma: challenges and opportunities. Methods Mol Med 2005; 113: 1-4.

5. Seidel C, Hjertner O, Abildgaard N, et al. Serum osteoprotegerin levels are reduced in patients with multiple myeloma with lytic bone disease. Blood 2001; 98 2269-71.

6. Bataille R, Manolagas SC, Berenson JR. Pathogenesis and management of bone lesions in multiple myeloma. Hematol Oncol Clin North Am 1997; 11: 349-61.

7. Gregory CA, Gunn WG, Reyes E, et al. How Wnt signaling affects bone repair by mesenchymal stem cells from the bone marrow. Ann N Y Acad Sci 2005; 1049: 97-106.

8. Glass DA, Patel MS, Kassenty G. A new insight into the formation of osteolytic lesions in multiple myeloma. N Engl J Med 2003; 349: 2479-80.

9. Vanderkerken K, Asosingh K, Croucher P, et al. Multiple myeloma biology: lessons from the 5TMM models. Immunol Rev 2003; 194: 196-206.

10. Roodman GD. Pathogenesis of myeloma bone disease. Blood cells Mol Dis 2004; 32: 290-2.

11. Kelley SK, Ashkenazi A. Targeting death receptors in cancer with Apo2L/TRAIL, Curr Opin Pharmacol 2004; 4: 333-9.

12. Gazitt Y. TRAIL is a potent inducer of apoptosis in myeloma cells derived from multiple myeloma patients and is not cytotoxic to hematopoietic stem cells. Leukemia 1999; 13 1817-24.

13. Chen Q, Ray S, Hussein MA, et al. Role of Apo2L/TRAIL and $\mathrm{BCl}$-2-family proteins in apoptosis of multiple myeloma. Leuk Lymphoma 2003; 44: 1209-14.

14. Mitsiades N, Mitsiades CS, Poulaki V, et al. Concepts in the use of TRAIL/Apo2L: an emerging biotherapy for myeloma and other neoplasias. Expert Opin Invest Drugs 2001; 10: 1521-30.

15. Brunetti G, Oranger A, Mori G, et al. TRAIL is involved in human osteoclast apoptosis. Ann N Y Acad Sci 2007; 1116 316-22.

16. Brunetti G, Colucci S, Rizzi R, et al. The role of OPG/TRAll complex in multiple myeloma: the OPG/TRAIL complex in an in vitro osteoclastogenesis model derived from human multiple myeloma-bone disease. Ann N Y Acad Sci 2006; 1068: 334-40.

17. Fu Jin-Xiang, Shi Xiaofenga, Qin Jun-Chuanc, et al. Homing efficiency and hematopoietic reconstitution of bone marrow-derived stroma cells expanded by recombinant human macrophage-colony stimulating factor in vitro. Exp Hematol 2004; 32: 1204-11.

18. Giuliani N, Rizzoli V, Roodman GD. Multiple myeloma bone disease: pathophysiology of osteoblast inhibition. Blood 2006; 108: 3992-6.

19. Kyle RA, Rajkumar SV. Multiple myeloma. N Engl J Med 2004; 351: 1860-73.
20. Robbiani DF, Chesi M, Bergsagel PL. Bone lesions in molecular subtypes of multiple myeloma. N Engl J Med 2004; 351: 197-8.

21. Yaccoby S, Pearse RN, Johnson CL, et al. Myeloma interacts with the bone marrow microenvironment to induce osteoclastogenesis and is dependent on osteoclast activity. Br J Haematol 2002; 116: 278-90.

22. Vejlgaard T, Abildgaard $\mathrm{N}$, Jans $\mathrm{H}$, et al. Abnormal bone turnover in monoclonal gamyelomaopathy of undetermined significance: analyses of type I collagen telopeptide, osteocalcin, bone-specific alkaline phosphatase and propeptides of type I and type III procollagens. Eur J Haematol 1997; 58: 104-8.

23. Silvestris F, Cafforio P, Tucci M, et al. Upregulation of osteoblast apoptosis by malignant plasma cells: a role in myeloma bone disease. Br J Haematol 2003; 122: 39-52.

24. Tinhofer I, Biedermann R, Krismer M, et al. A role of TRAIL in killing osteoblasts by myeloma cells. FASEB J 2006; 20: 751-6.

25. Giuliani N, Rizzoli V, Roodman GD. Multiple myeloma bone disease: pathophysiology of osteoblast inhibition. Blood 2006; 108: 3992-6.

26. Gregory CA, WG Gunn, Reyes E, et al. How wnt signaling affects bone repair by mesenchymal stem cells from the bone marrow. Ann NY Acad Sci 2005; 1049: 97-106.

27. Gunn WG, Conley A, Deininger L, et al. A crosstalk between myeloma cells and marrow stromal cells stimulates production of DKK1 and interleukin-6: a potential role in the development of lytic bone disease and tumor progression in multiple myeloma. Stem Cells 2006; 24: 986-91.

28. Tian E, Zhan F, Walker R, et al. The role of the Whtsignaling antagonist DKK1 in the development of osteolytic esions in multiple myeloma. N Engl J Med 2003; 349: 2483-94.

29. Arnult B, Lecourt S, Soulier J, et al. Phenotypic and functional characterization of bone marrow mesenchymal stem cells derived from patients with multiple myeloma. Leukemia 2007; 21: 158-63.

30. Calvani N, Cafforio P, Silvestris F, et al. Functional osteoclast-like transformation of cultured human myeloma cell lines. Br J Haematol 2005; 130: 926-38.

31. Shipman CM, Croucher PI. Osteoprotegerin is a soluble decoy receptor for tumor necrosis factor-related apoptosisinducing ligand/Apo2 ligand and can function as a paracrine survival factor for human myeloma cells. Cancer Res 2003; 63: 912-6. 